\title{
Methodologische Grundlegung und methodisches Vorgehen
}

Das folgende Kapitel zielt auf eine forschungspraktische Darstellung und methodologische Reflexion des eigenen Forschungsprozesses. Es wird ein Überblick über die verschiedenen Forschungsschritte geliefert und die dabei zur Anwendung gekommenen forschungspraktischen Strategien. $\mathrm{Zu}$ beachten ist hier, dass die folgend beschriebenen Schritte keine fest im Voraus geplanten Forschungsschritte darstellen, sondern sich in einer Ko-Konstruktion zwischen Forschungsinteresse und Untersuchungsgegenstand während der Forschung konkretisiert haben. Zudem stellt die dargestellte Differenzierung der verschiedenen Forschungsschritte eine analytische Unterscheidung dar, welche in der Forschungspraxis nicht in dieser strikten Trennung ablief. Eine solche fehlende strikte Orientierung an fest abfolgenden Forschungsschritten ist typisch für pragmatische Forschungsdesigns (Bogusz 2013; Dewey 2008; Strauss 1998).

Die folgende Darstellung soll einen Einblick gewähren in die Forschungspraxis, der die in den folgenden Kapiteln dargestellten Resultate entsprungen sind. Es soll jedoch nicht lediglich auf arbeitsorganisatorische Fragen eingegangen werden, sondern einem methodischen Holismus folgend auch (Diaz-Bone 2010c, S. 183 ff.), wie methodologisch versucht wurde, die Theorieperspektive an das Datenmaterial heranzubringen und an dieses zu vermitteln (Reichertz 2009, S. 279). Eine Herausforderung besteht hierbei darin, dass in der EC kein fester Kanon an Forschungs- und Auswertungsstrategien existiert (Diaz-Bone 2018, S. 389). Auf der Basis verschiedener sozialwissenschaftlicher Methoden wird deshalb im Folgenden eine Erhebungs- und Auswertungsstrategie ausgearbeitet, welche es aus methodologischer Perspektive ermöglichen soll, unterschiedliche Logiken der Survey-Produktion rekonstruieren zu können.

$\mathrm{Zu}$ Beginn der Forschung musste zunächst ein Überblick über den Forschungsgegenstand erarbeitet werden. Hierzu wurden Interviews mit 
Survey-Methodologen und Survey-Praktikern geführt. Identifiziert wurden hier zunächst Survey-Projekte und Situationen der Survey-Koordination, welche sich gemäß dem Konzept des theoretischen Sampelns für weiterführende Erhebungen eignen (vgl. ausführlich Abschn. 4.2). Zugleich fand während dieses Schritts eine Konkretisierung des Forschungsinteresses statt. Während den ersten empirischen Erhebungen und nachfolgenden Analysen wurde deutlich, dass sich die Theorie der Produktionswelten als Erklärungsmodell für die bei Surveys vorzufindenden Unterschiede der Survey-Produktion eignet (Storper und Salais 1997). Es zeigte sich hierbei, dass die Theorie der Produktionswelten fähig ist, einerseits die zu diesem Zeitpunkt identifizierten Unterschiede aufnehmen und erklären zu können und andererseits den Fokus auf noch nicht analysierte Unterschiede in der Survey-Produktion zu lenken und dort ebenfalls analytisches Potenzial zu entfalten. ${ }^{1}$

\subsection{Methodologische Grundlagen}

Methodologisch ist die EC aus verschiedenen Gründen schwierig einzuordnen. Einerseits finden sich methodologische Hinweise nur vereinzelt in den Gründungstexten (Diaz-Bone 2011b, S. 45). Andererseits positionieren sich die verschiedenen Vertreter der EC methodologisch unterschiedlich, bzw. werden als unterschiedlich positioniert wahrgenommen. ${ }^{2}$ Rainer Diaz-Bone sieht in der EC keinen reinen methodologischen Individualismus. Die methodologische Position der EC sieht er gerade durch eine Überwindung der Gegensätze zwischen

${ }^{1}$ Ein Beispiel stellt die in Abschn. 5.4 dargestellte Informationswelt dar, welche der industrieweltlichen Produktionswelt bei Salais und Storper entspricht (1997, S. 32 ff.). Vor dem analytischen Bezug auf die Theorie der Produktionswelten wurde stets die Trennung zwischen öffentlich-rechtlichen und privatwirtschaftlichen Survey-Projekten aufrechterhalten. Infolge der Produktionsweltentheorie wurde deutlich, dass diese Unterscheidung nicht zwingend ist und sich survey-weltlich nicht begründen lässt. Zentral ist für die Produktionsweltentheorie nicht die institutionelle Einbettung, sondern die tatsächliche Koordinationspraxis. Deswegen finden sich öffentlich-rechtliche und privatwirtschaftliche Survey-Projekte gleichermaßen in der Informationswelt wieder. $\mathrm{Zu}$ unterschieden sind Projekte hier aber nach der Reichweite der produzierten Daten und durch die Perspektive der Rechtfertigungsordnungen lassen sich in einem zweiten Schritt wieder grundlegende Unterschiede zwischen den verschiedenen institutionellen Einbettungen aufzeigen (Boltanski und Thévenot 2007).

${ }^{2}$ Hierbei ist insbesondere die Diskussion um den methodologischen Individualismus von Bedeutung. Latsis sieht in der EC keinen starken methodologischen Individualismus (2006, S. 266). Diese Position wird von Thévenot mit Bezug auf Diaz-Bone geteilt (2015, S. 203). 
methodologischem Individualismus und Holismus charakterisiert an (Diaz-Bone 2018, S. 378 ff.). ${ }^{3}$

Der methodologische Standpunkt der EC greift stark auf die Arbeiten der Pragmatisten William James und John Dewey zurück (Diaz-Bone 2018, S. 332 ff.). Zentral ist dabei das Konzept des methodologischen Experimentalismus (Bogusz 2013). Sozialwissenschaftliche Theorien sind in einer solchen Perspektive nicht die Bedingung für Erkenntnis, sondern die Grundlagen dazu. Das Forschungsobjekt wird konstruiert, die Konstruktion soll jedoch durch das Forschungsobjekt irritiert werden (Kalthoff 2008, S. 21). Das Forschungsobjekt wird so konstruiert, ohne dass damit eine Performativität der Theorie am Forschungsobjekt einhergehen würde. Mit Dewey kann darauf verwiesen werden, dass die deduktiv-hypothetische Forschung lediglich einen Zwischenschritt im Forschungsprozess darstellt. Vorlaufend ist die (induktive) Entwicklung von Hypothesen (Dewey 2008, S. 493). ${ }^{4}$ Die forschungslogische Position Deweys und damit die zentrale Grundlegung des methodologischen Experimentalismus lässt sich in drei Punkten zusammenfassen:

1. Der Status theoretischer Begriffe entspricht Hypothesen ${ }^{5}$

2. Diese Hypothesen haben eine lenkende Funktion bei der Kontrolle der Beobachtung

3. Die Hypothesen werden im (empirischen) Forschungsprozess überprüft und kontinuierlich revidiert (Dewey 2008, S. 583)

Zentral ist es aus der Perspektive des methodologischen Experimentalismus folglich, die eingenommene theoretische Perspektive während des Forschungsprozesses konstant zu überprüfen und gegebenenfalls zu revidieren. Im Folgenden werden die für diese Arbeit relevanten methodologischen Grundlegungen der EC aufgearbeitet werden.

Die Situation stellt den zentralen methodologischen Bezugspunkt der EC dar (Boltanski und Thévenot 2007, S. 11 ff.; Diaz-Bone 2011b, S. 47; Wagner

\footnotetext{
${ }^{3}$ Mohamed Nachi fragt in seinem Einführungsbuch $\mathrm{zu}$ den entstehenden pragmatischen Sozialwissenschaften in Frankreich bereits im Untertitel, ob es sich hier um die Entwicklung eines neuen soziologischen Stils handelt (2006).

${ }^{4}$ Vgl. zu dieser Position auch Hirschauer (2008, S. 168 ff.).

${ }^{5}$ Theorien sind in der Tradition des Pragmatismus folglich ,sensiblen Konzepten“ der Grounded Theory nicht unähnlich (Strübing 2008, S. 57 ff.). Nicht überraschend vertritt diese Position auch Herbert Blumer (2004, S. 350 f.).
} 
1993, S. 465). ${ }^{6}$ Das Situationskonzept ist in der EC jedoch breit gefasst und darf nicht auf face-to-face-Situationen reduziert werden. Situationen können in der EC eine hohe raum-zeitliche Ausdehnung einnehmen (Diaz-Bone 2018, S. 374 ff.). Elemente einer Situation sind Akteure, Objekte, Formen und Konventionen. Rekonstruiert werden die verschiedenen Elemente einer Situation und deren Vernetzung und Mobilisierung. Diese Konstellationen werden aus der subjektiven Sicht der involvierten Akteure rekonstruiert, das heißt, es ,wird eine pragmatische Innenansicht der Situation angestrebt" (Diaz-Bone 2018, S. 330). Diese Innensicht wird eingenommen, um die Situation als solche eingrenzen zu können. Es gilt diejenige zeitliche und räumliche Ausdehnung zu beachten, welche in der Situation selbst mobilisiert wird (Dodier 2011, S. 71). Die Situation als Untersuchungsobjekt lässt sich folglich erst während der empirischen Forschung bestimmen. Empirische Arbeiten der EC fokussieren vor allem auf Konfliktsituationen. Denn hier ist beobachtbar, wie Kontingenzen bewältigt und eine allgemeine Ordnung in der Situation wiederhergestellt wird (Boltanski und Thévenot 2007, S. 181).

Die EC unterscheidet nicht zwischen Mikro-, Meso- und Makroebene. Das Ebenenkonzept wird ersetzt durch ein Reichweitenkonzept von situativen Koordinationsformen. Unterschieden wird folglich nicht zwischen Koordinationsformen auf der Mikro- und der Makroebene, sondern zwischen unterschiedlichen Reichweiten, welche Koordinationsformen sozio-geografisch einnehmen (Diaz-Bone 2018, S. 375). Dies ist der Grund für die methodologische Fokussierung auf Situationen, da in ihr Situationselemente mit unterschiedlicher Reichweite integriert werden. Die Zuschreibung von Kausalität wird in der EC nicht auf menschliches Handeln reduziert. Vielmehr wird in der Folge eines methodologischen Situationalismus Handlung als Ko-Konstruktion von Akteuren, Konventionen und Objekten verstanden. Handlung lässt sich folglich nicht auf individuelle Absichten reduzieren, sondern muss auf die restlichen Situationselemente bezogen werden. In Bezug auf die Miteinbeziehung von Objekten teilt die EC folglich die Position einer verallgemeinerten Symmetrie (Latour 2008, S. 127 ff.) der ANT (Diaz-Bone 2018, S. 370). ${ }^{7}$ Die Einführung dieser

\footnotetext{
${ }^{6}$ Und folglich nicht Gesellschaft oder die Gesellschaft.

${ }^{7}$ Hier kann kritisch angefragt werden, inwiefern das Objektkonzept der EC und der ANT vergleichbar ist. Die ANT bezieht sich explizit auf biologische, physikalische und chemische Eigenlogiken von Objekten. Ein Beispiel ist hier das Fortpflanzungsverhalten von Jakobsmuscheln in der St. Brieuc-Bucht in Frankreich (Callon 2006). Im Gegensatz dazu interessieren sich beispielsweise Boltanski und Thévenot nicht für materiale Eigenlogiken, sondern für soziale Signifikationsprozesse von Objekten. Denn Objekte sind in „Über die
} 
verallgemeinerten Symmetrie stellt einen Bruch mit der durkheimschen Soziologie im Hinblick auf die Bedeutung einer materialen Umwelt für soziales Handeln dar (Latour 2008, S. 72). ${ }^{8}$ In Abgrenzung zur bourdieuschen Soziologie werden Handlungs-, bzw. Koordinationsprodukte in der EC nicht auf inkorporierte Dispositionen zurückgeführt (Diaz-Bone 2011b, S. 53; Thévenot 2011, S. 282, 256; Thévenot 2011d, S. 256).

Ein zentraler Abgrenzungspunkt stellte für die EC die Sozialtheorie Bourdieus dar. Kritisiert wurde daran die Reduktion der vielfältigen gesellschaftlichen Strukturierungsprinzipien auf das Prinzip des sozialen Raums und die verschiedenen Kapitalien durch Bourdieu. An die Stelle der Rückführung auf eine Struktur - die des sozialen Raums - tritt in der EC in Anlehnung an den Pragmatismus eine Pluralität von strukturierenden Logiken (Diaz-Bone 2018, S. 380 ff.). Die EC postuliert hier eine doppelte Pluralität ${ }^{9}$ (Diaz-Bone 2011a, S. 29), wobei die horizontale Pluralität die Vielfalt der Rechtfertigungsordnungen darstellt und die vertikale Pluralität die verschiedenen Handlungsregime. Zentral ist hier die Maxime, ein Handlungsprinzip nicht durch ein anderes zu erklären

Rechtfertigung“ als Stützen der Handlung gedacht (Boltanski und Thévenot 2007, S. 34), deren Tragfähigkeit - anders als im Beispiel der Jakobsmuscheln - nicht von biologischen, physikalischen oder chemischen Faktoren abhängt, sondern von der Reichweite der Signifikationsprozesse. In Anlehnung an Thévenots Theorie der Formen stellt sich dann die Frage, ob Objekte in der EC nicht eher materiell abgestützte Formen darstellen.

${ }^{8}$ Die Kritik von Latour am Strukturalismus bezieht sich dabei auch auf die französische Epistemologie, welche insbesondere durch Gaston Bachelard (1987), Georges Canguilhelm (2001) und Pierre Bourdieu (1991, 1992) entwickelt und weiterentwickelt wurde. Die Kritik bezieht sich dabei darauf, dass auch die französische Epistemologie eine Trennung zwischen Subjekt und Objekt, sowie Natur und Gesellschaft aufrecht erhalte (Latour 2007, 2008). Wie Mary Tiles mit Bezug auf die Arbeiten von Bourdieu jedoch aufzeigt, vermischt Latour dabei verschiedene Konzeptionen des epistemologischen Diktums der Unabhängigkeit und Autonomie von Wissenschaft (Tiles 2011). Die Unabhängigkeit der Wissenschaft im Hinblick auf ihre Problemstellungen sollte nicht mit der auch von Bourdieu kritisierten "scholastischen Vernunft“ verwechselt werden (Bourdieu 2004). Die von Latour vorgenommene Kritik an der Epistemologie verkennt folglich die Autonomie der eigenen Wissenschaftsposition von gesellschaftlichen und auch weiteren wissenschaftlichen Diskursen und einen reflexiven Zugang zur eigenen Epistemologie.

${ }^{9}$ Wobei eine doppelte Pluralität nicht nur bei Thévenot durch seine Begründung der Theorie der Regime des Engagements zu finden ist, sondern auch bei Boltanski in Form seiner Regimetheorie (Boltanski 2012, S. 68 ff.) und bei Favereau infolge einer Differenzierung des Konventionenkonzepts (Favereau 2008). 
(Benatouil 1999, S. 383). Zentral sind in den Analysen der EC jedoch nicht nur die Identifikation von Konventionen als Koordinationsgrundlagen, sondern insbesondere auch deren pragmatische Durchsetzung und Abstützung (Diaz-Bone 2018, S. 371 ff.). Die EC interessiert sich folglich nicht lediglich für eine Kartografierung und eine Identifikation der empirisch feststellbaren Koordinationsformen, sondern gerade auch für deren gegenseitige Kritik und Konflikte zwischen den verschiedenen Rechtfertigungsordnungen (Boltanski und Thévenot 2007, S. 287 ff.), ${ }^{10}$ für deren historische Entwicklung (Boltanski und Chiapello 2006) und deren räumliche Reichweite (Lamont und Thévenot 2000). ${ }^{11}$ In der Forschungspraxis zeigt sich die Berücksichtigung der Vielfalt von strukturierenden Prinzipien in einem reflexiven Sprachgebrauch bei der Beschreibung von Koordinationslogiken. Boisard weist beispielsweise darauf hin, dass er mit einer alltäglichen Beschreibung des auf der häuslichen Rechtfertigungsordnung basierenden Herstellungsprozesses von traditionellem Käse unweigerlich eine Verfälschung der empirischen Begebenheiten in Richtung industrieller Rechtfertigungsordnung vornehmen würde (Boisard 1991, S. 173). Mit Foucault lässt sich formulieren, dass es deshalb bei der empirischen Arbeit zentral ist, die verschiedenen Koordinationslogiken in ihrem eigenen Drängen zu beschreiben (Foucault 2008, S. 498). ${ }^{12}$

\footnotetext{
${ }^{10}$ Der Fokus auf Probleme der Übersetzung zwischen Koordinationsformen zeigt sich insbesondere auch in der Regimetheorie. Thévenot verweist beispielsweise auf das Problem der Übersetzung zwischen verschiedenen Handlungsregimen bei der Pflegearbeit (Thévenot 2009b, S. 808). In neueren Forschungen wird beispielsweise auf die Artikulationsprobleme von sozialen Bewegungen als Regimekonflikten hingewiesen (Blok und Meilvang 2015; Conley und Jensen 2016; Thévenot 2014b).

${ }^{11}$ Und genau hierin liegt einer der methodologischen Unterschiede zur foucaultschen Diskursanalyse, indem die Konventionentheorie die Dynamik von Koordinationslogiken $\mathrm{zu}$ identifizieren und beschreiben versucht und so die pragmatische Seite von Strukturen betont. Ein Beispiel für eine solche Studie ist neben der erwähnten Studie von Boltanski und Chiapello die Studie von Salais et al. zur Entstehung der Kategorie der Arbeitslosigkeit (Salais et al. 1999). Im Gegensatz dazu vertritt Foucault den Standpunkt, dass der Ursprung von Diskursen nicht identifiziert werden kann: „Das eine verlangt, dass es nie möglich ist, in der Ordnung des Diskurses den Einbruch eines wirklichen Ereignisses zu bestimmen; dass jenseits jedes offenbaren Beginns es stets einen geheimen Ursprung gibt - einen so geheimen und so ursprünglichen, dass man nie ihn völlig in sich selbst erfassen kann“ (Foucault 2008, S. 497).

${ }^{12}$ Die Konventionentheorie vertritt damit einen vergleichbaren methodologischen Standpunkt zur foucaultschen Diskursanalyse, indem sie eine Koordinationslogik in ihrem „So-Sein“ analysieren und nicht auf „die ferne Präsenz des Ursprungs verweisen“ will (Foucault 2008, S. 498). Hierbei zeigt sich jedoch ein theoretisches und methodologisches
} 
Der Vergleich stellt eine wichtige methodologische Strategie für die empirische Identifikation der verschiedenen Handlungslogiken der doppelten Pluralität der EC dar (Diaz-Bone 2015a, S. 325 f.). Hierbei zeigt sich der strukturalistische Einfluss der EC (Diaz-Bone 2018, S. 383). Die systematisch vergleichende Untersuchung verschiedener Produktionslogiken und/oder Fälle dient dabei dem Aufzeigen der Kontingenz der jeweiligen die Praxis anleitenden Konventionen (Diaz-Bone 2015a, S. 320). ${ }^{13}$ Denn erst durch den systematischen Vergleich wird sichtbar, dass das So-Sein des Untersuchungsgegenstandes nicht naturgemäß in dieser Form besteht, sondern ein Ergebnis des strukturierenden Einflusses einer Konvention darstellt. Die Strategie des systematischen Vergleichs dient folglich dazu, die natürliche Angemessenheit von Handlungen infrage zu stellen und den Blick zu öffnen für deren Strukturierung durch Konventionen.

Konventionen stellen nicht nur idealtypische Rekonstruktionen von Sozialforschern dar, sondern sind auch idealtypische Stützen für die Handlung von Akteuren (Diaz-Bone 2011b, S. 55; Storper und Salais 1997, S. 27). Sie sind vielmehr das grundlegende Sinnprinzip, auf dem aufbauend Akteure Regeln, Vorgaben, Hinweise etc. situativ interpretieren. Sowohl Sozialforscher wie auch Akteure orientieren sich folglich an einer Ebene, welche Regeln vorausgeht und damit Regeln in deren Funktion als strukturbildendes Element erst ermöglicht. Gerade diese fehlende Regelbasierung macht Konventionen zu tacit knowledge

Grundproblem der Konventionentheorie, dass sie einerseits Konventionen in der Nachfolge von Lewis als Grundlage für ,handfeste“ Koordinationsprobleme verstehen will (Lewis 1975, S. 41 ff.). Diese Interpretation würde gerade den Bezug auf die von Foucault angesprochene „ferne Präsenz des Ursprungs“ (Foucault 2008, S. 498) ermöglichen und motivieren. Dagegen ist die Konzeptualisierung von Konventionen als Rechtfertigungsordnungen mit dem Konzept der Diskursordnung insoweit vergleichbar, als dass sie die handlungspragmatische Dimension von Koordinationsproblemen übersteigt und zusätzlich die moralische Frage nach dem Gemeingut und der moralischen Grundlage der gemeinsamen Sache einbringt (Boltanski und Thévenot 2007, S. 60 ff.).

${ }^{13}$ Und hierin besteht folglich eine vergleichbare methodologische Strategie zur bourdieuschen Soziologie, wie im folgenden Zitat von Bourdieu et al. deutlich wird: „Kurzum, der Vergleich auf der Grundlage der Hypothese von Analogien ist nicht nur das wichtigste Instrument für den Bruch mit den präkonstruierten Gegebenheiten, die immer wieder an und für sich selbst behandelt werden wollen; er ist vielmehr auch Grundlage der hypothetischen Konstruktion von Beziehungen zwischen den Beziehungen“ (Bourdieu et al. 1991, S. 60). Die Strategie des Vergleichs dient in beiden soziologischen Theorierichtungen dem Aufzeigen von hinter den „Erscheinungen“ des Untersuchungsgegenstandes liegenden und diese strukturierenden Logiken. 
(Collins 2010, S. 121 ff.). Die Eigenschaft als tacit knowledge macht deren formelle (Wissens-) Weitergabe unmöglich (Collins 2010, S. 129) und ermöglicht den von der EC angenommenen fähigen Akteur. ${ }^{14}$

\subsection{Datenerhebung}

Die folgenden Ausführungen thematisieren die Datenerhebung, d.h. die Erstellung des Datenkorpus, welcher schlussendlich als Basis für die Datenanalyse diente. Um Anhaltspunkte für Situationen zu erhalten, in welchen sich Unterschiede in der Koordination in Survey-Projekten zeigen, war es zunächst notwendig, sich eine Wissensbasis über die Survey-Forschung zuzulegen. Dies geschah einerseits über das Einlesen in die Survey-Methodologie, um Strategien und Probleme der Survey-Forschung aus der Theorieperspektive ${ }^{15}$ vertiefen zu können. Um einen Einstieg in die Thematik der praktischen Koordination in Survey-Projekten zu erhalten, wurden Experteninterviews mit verschiedenen Survey-Praktikern durchgeführt. In Anlehnung an das Konzept des Theoretical Samplings der Grounded Theory wurde auf der Basis der durchgeführten Interviews eine konstante Detaillierung der Forschungsfragen vorgenommen (Strauss 1998, S. 70 f.). Dies führte dazu, dass mit zunehmender Anzahl der getätigten Interviews eine Verschiebung des Explorationscharakters der Interviews hin zu einer vertieften Auseinandersetzung mit dem Gegenstand stattfand, das heißt zu einer direkten Klärung von survey-weltlichen Fragen. Insgesamt wurden elf Interviews durchgeführt. Der Expertenstatus wurde den Interviewten durch ihre langjährige praktische Erfahrung in der Durchführung von Survey-Projekten auf Auftragnehmer- oder Auftraggeberseite zugeschrieben (Gläser und Laudel 2010, S. 12; Meuser und Nagel 2009, S. 37 f.). Von den elf effektiv durchgeführten Interviews wurden schlussendlich sieben komplett für die Analyse transkribiert und

\footnotetext{
${ }^{14}$ Hier lässt sich wiederum auf das von Favereau formulierte zentrale Axiom der Konventionentheorie verweisen, wonach die Unvollständigkeit von Regeln nicht ein Problem, sondern gerade die Lösung für deren Koordinationsfunktion ist (Favereau 1989a, S. 295). Erst die Unvollständigkeit von Regeln erlaubt deren sinnhafte Anwendung über verschiedenen Situationen hinweg. Der Versuch einer alleinig formalen Bestimmung der Anwendung einer Regel würde unweigerlich an den höchst unterschiedlichen Situationsspezifitäten scheitern. Es ist das Sinnprinzip von Konventionen, welches im Gegenzug formale Regeln auf eine Vielzahl von Situationen beziehbar macht.

${ }^{15}$ Als Theorie des Untersuchungsgegenstandes, welche als Informationsquelle über den Untersuchungsgegenstand diente, jedoch nicht als forschungsleitende Theorie.
} 
analysiert. Die anfänglichen Experteninterviews stellten die Grundlage für die Fallauswahl und die ethnographischen Beobachtungen dar. Es wurden jedoch auch noch während der Zeit der Durchführung der ethnografischen Beobachtungen Interviews geführt, welche oft direkt auf das Beobachtete bezogen waren.

Sämtlichen interviewten Personen wurde Anonymität zugesichert, sodass bei Zitaten Firmen-, Personennamen und weitere Hinweise auf die interviewte Person gestrichen werden, wie auch deren genaue Funktion. Zudem wurde durch die dem Interview vorhergehende Darstellung des Forschungsprojekts versucht, eine informierte Zusage zu gewährleisten, d. h. die interviewten Personen wurden über den Sinn und Zweck der Interviews sowie deren Verwendung aufgeklärt (Helfferich 2009, S. 190). Bei der Anonymisierung von Forschungsdaten muss grundlegendend ein Kompromiss zwischen zwei Zielen eingegangen werden (Gebel et al. 2015). Einerseits existiert auch in qualitativ ausgerichteten Arbeiten die Notwendigkeit, Aussagen und Schlussfolgerungen für Lesende möglichst nachvollziehbar zu gestalten. Dafür ist es notwendig, möglichst viele Hintergrundinformationen zur Verfügung zu stellen. Auf der anderen Seite besteht die Verpflichtung zum Datenschutz durch Anonymisierung, um das forschungsethische Credo einhalten zu können, wonach für interviewte Personen durch das Interview kein Nachteil entstehen darf (Kretzer 2013, S. 3). Diese Problematik stellt sich auch bei der vorliegenden Arbeit. Sie wird dadurch noch verstärkt, dass das Survey-Feld in der Schweiz ziemlich überschaubar ist. Der Verweis darauf, dass ein Interview beispielsweise mit der geschäftsleitenden Person einer größeren Survey-Firma geführt wurde, hätte den Effekt, dass insgesamt maximal zwei bis drei Personen dafür infrage kommen würden. Durch das Beiziehen weiterer Kontextinformationen aus dem entsprechenden Interview wäre in der Folge relativ schnell klar, um welche Person es sich handeln muss (Unger 2014, S. 25). Aus diesem Grund wurde nicht nur eine formale Anonymisierung vorgenommen, sondern es wurde eine faktische Anonymisierung angestrebt (Gebel et al. 2015, S. Abs. 25 ff.). Dies hat für die vorliegende Arbeit Konsequenzen. So wurde darauf verzichtet, die jeweiligen Interviewzitate einer - wenn auch fiktiven - Person zuzuordnen, da eine faktische Anonymisierung durch die Möglichkeit des Zusammenfügens verschiedener Interviewausschnitte bereits nicht mehr gegeben wäre. Stattdessen wird jeweils direkt bei den Zitaten eine Charakterisierung der Person vorgenommen, soweit dies für die Einordnung der getätigten Aussagen notwendig ist.

Die ethnografischen Beobachtungen waren einerseits orientiert an der Analyse von Survey-Welten und andererseits von Survey-Projekten. Für die Erreichung des ersten Ziels wurden bereits früh ethnografische Beobachtungen 
an einer Generalversammlung des VSMS-Verbandes ${ }^{16}$ getätigt. Danach wurden Koordinationssitzungen zwischen verschiedenen Auftraggebern und verschiedenen Survey-Firmen teilnehmend beobachtet. Den zweiten Teil der ethnografischen Arbeit stellten Beobachtungen bei zwei verschiedenen Survey-Firmen dar, in welchen der reguläre Tagesablauf von je einer als Projektleiter tätigen Person mitverfolgt wurde. Diese Beobachtungen wurden durch das Mitverfolgen von telefonischen Interviews während Abendschichten in einer Survey-Firma ergänzt, um einen Eindruck von der Befragungspraxis bei telefonischen Interviews gewinnen zu können.

Ethnographische Beobachtungen dienten jedoch auch als Grundlage für die beiden Fallanalysen. So wurde in Fall (A) die zentrale Koordinationssitzung zwischen der Survey-Firma und dem Auftraggeber beobachtet und ebenfalls audiotechnisch aufgenommen. In Fall (B) wurde einerseits die zentrale Koordinationssitzung zwischen einem Hotline-Dienstleister und dem Auftraggeber beobachtet und ebenfalls audiotechnisch aufgenommen, andererseits wurde die Briefing-Sitzung und die De-Briefing-Sitzung zwischen Survey-Firma und Auftraggeber beobachtet, jedoch ohne audiotechnische Aufnahmen. Sowohl in Fall (A) und (B) wurde je ein zusätzliches Interview mit den Leitern des Survey-Projekts auf Auftraggeberseite durchgeführt und es wurde mittels verschiedener Projektdokumente (Offerten, Verträge, Berichte der Survey-Firma und des Auftraggebers etc.) ein Dokumentenkorpus zu den beiden Fällen erstellt. In der folgenden Tabelle ist die empirische Grundlage der Forschung zusammenfassend aufgeführt. $\mathrm{Zu}$ beachten ist, dass die verschiedenen Datenerhebungen nicht zwingend in dieser Reihenfolge zustande kamen. In Tab. 4.1 sind die Forschungsschritte und die daraus resultierende Datengrundlage tabellarisch aufgeführt.

Die Datenerhebung geschah innerhalb eines durch den SNF geförderten Projekts. Aus diesem Grund wurden zwei Experteninterviews zu dritt und die restlichen zu zweit mit weiteren projektbeteiligten Personen geführt. So wurden auch sämtliche Beobachtungen, bis auf einen Tag Beobachtung bei der SurveyFirma (1) und die zwei Tage Beobachtung bei der Survey-Firma (2), zu zweit durchgeführt. Auch wurde ein gemeinsamer Korpus an Dokumenten im Hinblick auf die beiden Fälle angelegt.

\footnotetext{
${ }^{16}$ Der Verband Schweizer Markt- und Sozialforschung stellt die Interessenvereinigung der Schweizer Markt- und Sozialforschungsinstitute und deren Auftraggeber dar (VSMS 2017).
} 
Tab. 4.1 Die Datenerhebung im Überblick

\begin{tabular}{|c|c|}
\hline Forschungsschritt & Datengrundlage \\
\hline Explorative Experteninterviews & $\begin{array}{l}\text { Elf Experteninterviews mit } \\
\text { Survey-Methodologen und Survey-Prakti- } \\
\text { kern }\end{array}$ \\
\hline \multirow[t]{4}{*}{ Explorative ethnografische Beobachtungen } & Beobachtung VSMS-Generalversammlung \\
\hline & $\begin{array}{l}\text { Drei Tage ethnografische Beobachtung von } \\
\text { Arbeitsprozessen der Survey-Produktion bei } \\
\text { Survey-Firma (1), inklusive Beobachtung } \\
\text { einer Sitzung mit einem Auftraggeber }\end{array}$ \\
\hline & $\begin{array}{l}\text { Zwei Tage ethnografische Beobachtung bei } \\
\text { Survey-Firma (2) }\end{array}$ \\
\hline & $\begin{array}{l}\text { Zwei Abende Beobachtung und Mithören } \\
\text { von standardisierten telefonischen Inter- } \\
\text { views (CATI) bei Survey-Firma (1) }\end{array}$ \\
\hline \multirow[t]{3}{*}{ Fall $(A)$} & $\begin{array}{l}\text { Interview mit dem Leiter (Auftraggeber) des } \\
\text { Survey-Projekts zum Survey-Prozess von } \\
\text { Fall (A) }\end{array}$ \\
\hline & $\begin{array}{l}\text { Beobachtung der zentralen Koordinations- } \\
\text { sitzung zwischen Survey-Firma und Auf- } \\
\text { traggeber }\end{array}$ \\
\hline & $\begin{array}{l}\text { Verschiedene Dokumente aus dem Sur- } \\
\text { vey-Projekt }\end{array}$ \\
\hline \multirow[t]{5}{*}{ Fall $(B)$} & $\begin{array}{l}\text { Interview mit dem Leiter (Auftraggeber) des } \\
\text { Survey-Projekts zum Survey-Prozess von } \\
\text { Fall (B) (zugleich auch Experteninterview) }\end{array}$ \\
\hline & $\begin{array}{l}\text { Beobachtung der zentralen Koordinations- } \\
\text { sitzung mit einem Hotline-Dienstleister }\end{array}$ \\
\hline & $\begin{array}{l}\text { Beobachtung der Briefing-Sitzung zwischen } \\
\text { Auftraggeber und Survey-Firma }\end{array}$ \\
\hline & $\begin{array}{l}\text { Beobachtung der De-Briefing-Sitzung zwi- } \\
\text { schen Survey-Firma und Auftraggeber }\end{array}$ \\
\hline & $\begin{array}{l}\text { Verschiedene Dokumente aus dem Sur- } \\
\text { vey-Projekt }\end{array}$ \\
\hline
\end{tabular}

Im Folgenden werden die methodologischen Grundlagen der ethnografischen Beobachtungen aufgearbeitet und es wird geklärt, inwiefern diese zum Forschungsziel kompatibel sind. Ein zentraler Abgrenzungspunkt 
soziologisch-ethnographischer zu ethnologisch-ethnographischen Ansätze besteht in der Zurückweisung einer umfassenden Beschreibung von (Sub-) „Kulturen“. Hubert Knoblauch hat hier den Begriff der fokussierten Ethnografie geprägt (Knoblauch 2001). Die fokussierte Ethnografie geht von einer (vorläufigen) Bekanntheit des Gegenstandes aus, was durch die theoriegeleitete Konstruktion des Gegenstandes bedingt ist (Knoblauch 2001, S. 133 f.). ${ }^{17}$ Die Grundidee der fokussierten Ethnografie liegt darin, von gewissen Prozessen und Mechanismen im interessierenden Forschungsfeld zu abstrahieren, da sie aus der Perspektive der theoretischen Grundlegung des Forschungsprojekts nicht zentral für die Beantwortung der Forschungsfrage sind. Die Leistung einer soziologischen Ethnografie liegt folglich in einer Dekontextualisierung (Hirschauer 2001, S. 435). Zentral ist dabei die theoriegeleitete Konstruktion des Gegenstandes, sowie die theoriebasierte Begriffsbildung durch ethnografische Forschung (Atkinson 2015, S. 10 f.; Kalthoff 2008, S. 21). ${ }^{18}$ Die ethnografische Forschung im Sinne einer fokussierten Ethnografie versucht sich also nicht in einer möglichst umfassenden Beschreibung einer Kultur, sondern sie konzentriert sich auf die Herausarbeitung von zentralen und translokalen Mechanismen im Untersuchungsgegenstand. Dieses Herausarbeiten geschieht in Auseinandersetzung mit soziologischer Theorie. Ein zentrales Anliegen ethnografischer Forschung ist deswegen auch die Irritation bestehender Theoriesysteme (Breidenstein et al. 2013, S. 166).

Die Ethnografie, deren kleinster gemeinsamer Nenner die teilnehmende Beobachtung darstellt (Atkinson et al. 2001, S. 4; Breidenstein et al. 2013, S. 41), ${ }^{19}$ ist eine verhältnismäßig zeitaufwendige sozialwissenschaftliche Methode. Die

${ }^{17}$ Die fokussierte Ethnographie wendet sich dadurch gegen qualitativ-induktive Forschungsmethodologien wie die Grounded Theory, da sich diese gemäß Knoblauch nicht deutlich genug an Problemstellungen der soziologischen Theorie orientiere (Knoblauch 2001, S. 138).

${ }^{18}$ Atkinson und Hammersley kritisieren deswegen die Extended Case Method von Michael Burawoy, da diese suggeriere, dass andere ethnographische Ansätze in der Beschreibung von „Mikro“-Situationen steckengeblieben seien und nicht über das Partikulare hinauskommen würden (Atkinson 2015, S. 65). Wie Cicourel aufzeigte, wäre ein alleiniger Fokus auf Mikro-Situationen sinnlos, da immer Referenzen auf Situationen mit hoher Reichweite gemacht werden müssen (Cicourel 1981). Die Position von Atkinson und Hammersley verweist gerade auf die Untrennbarkeit von „Mikro“- und „Makro“-Situationen, was der methodologischen Position der EC infolge eines Denkens in Reichweiten anstelle von Ebenen entspricht (Diaz-Bone 2018, S. 375).

${ }^{19} \mathrm{Bzw}$. die beobachtende Teilnahme in der phänomenologischen Ethnographie (Honer und Hitzler 2015, S. 9 ff.). 
Begründung dafür, Verhalten minutiös zu beobachten und nicht „einfach“ Interviews durchzuführen, liegt in der Betonung der Differenz zwischen Sprache und Handeln (Gobo 2008, S. 5 f.). ${ }^{20}$ Die Ethnografie ist deswegen eine Methode, die Sachverhalte zur Sprache bringt, die vorher noch nicht Sprache waren (Hirschauer 2001). Die erklärungslogische Stoßrichtung ethnografischer Forschung besteht deswegen oft darin, eine Differenz zwischen Sprache und Handeln aufzuzeigen (Liebow 2003, S. 76 f.). ${ }^{21}$

Aus der Annahme einer Differenz zwischen Sprache und Handeln ergibt sich eine Kritik an einer lediglich auf Interviewdaten bezugnehmenden Sozialforschung (Jerolmack und Khan 2014a). Diese Kritik gründet darin, dass die Ethnografie die Einbettung von Handeln in soziale und materiale Umwelten betont. Kritisiert werden reine Interviewstudien deswegen als einem individualistischen Handlungskonzept unterliegend (Atkinson 2015, S. 13). Denn die Durchführung von akteurszentrierten qualitativen Interviews basiert auf der Annahme, dass sich soziale Prozesse durch den Fokus auf die Innensicht interviewter Personen erklären lassen. Dies schließt jedoch beispielsweise Routinen, Strukturierungsleistungen durch Objekte und Formen als Erklärungsleistungen aus. Ethnografien sind damit das Gegenteil von Experimenten, da gerade das Eingebettetsein in eine Umwelt als konstitutiv für menschliches Handeln stark gemacht wird (Knoblauch 2001, S. 134).

Eine ebenfalls situationszentrierte Perspektive nehmen die Workplace Studies ein. Aus ihrer Perspektive gehen klassische Ethnografien jedoch zu wenig weit, da sie das sequenzielle Herstellen von Situationen nicht in der notwendigen Detailliertheit nachzeichnen (Rawls 2008). Viele Arbeiten der Workplace Studies nehmen Bezug auf Konzepte der Ethnomethodologie (Luff et al. 2000a, S. 15). Die Workplace Studies betonen das „doing“ von Situationen. Diese sind folglich nicht durch Institutionen, Normen oder Regeln vordeterminiert, da auch deren spezifischer Sinn situativ hergestellt werden muss (Rawls 2008). Zwei Konzepte der Workplace Studies sind von besonderer Relevanz für die methodische Vorgehensweise der Datenerhebung und -auswertung dieser

\footnotetext{
${ }^{20}$ Bzw. in der Forderung einer stärkeren Theoretisierung dieses Übergangs, welcher durch Interviewmethoden oft als unproblematisch vorausgesetzt wird (Jerolmack und Khan 2014b).

${ }^{21}$ Ein berühmtes Beispiel hierfür stellt die Ethnographie „Talking about machines“ von Julian Orr dar, welcher die Differenz zwischen schriftlichen Anweisungen für die Reparatur von Kopiergeräten und der tatsächlichen handwerklichen Praxis aufzeigte (Orr 1996).
} 
Arbeit. Situationen sind aus der Perspektive der Workplace Studies durch eine grundlegende Reziprozität gekennzeichnet. Sie sind folglich nicht ausgestattete Orte, in welche Akteure eintreten und auf die gegebenen situationalen Ausstattungen Bezug nehmen können. Die Situation als grundlegende Analyseeinheit muss vielmehr als Produkt gegenseitiger Koordination verstanden werden. Referenzierte Objekte, Formen und Konventionen stellen so „oriented objects" dar, welche durch die in einer Situation anwesenden Akteure referenziert werden müssen, bevor sie als Situationsausstattung für die in einer Situation anwesenden Akteure verfügbar sind (Rawls 2018). Situationen sind dadurch das Ergebnis eines Koordinationsprozesses und nicht einfach gegeben. Im Hinblick auf die produktionsweltliche Grundlegung von Survey-Projekten bedeutet dies, dass Koordinationssituationen in Survey-Projekten ein Potential für verschiedene produktionsweltliche Koordinationslogiken aufweisen. Die spezifische produktionsweltliche Koordinationsform in Survey-Projekten muss also durch die verschiedenen Akteure aktiv durch das Referenzieren von produktionsweltspezifischen Objekten, Formen und Konventionen hergestellt werden und ergibt sich nicht etwa als logische Fortführung der Situationsausstattung selbst. Methodisch muss folglich der Prozesscharakter der Situationsprozessierung beachtet werden. Zentral ist deswegen die Beachtung des reflexiven Charakters von Situations-Koordination. Der Sinn von Situationen wird folglich durch die reflexive gegenseitige Bezugnahme der verschiedenen Akteure in einem konstanten Prozess kollektiv aufgebaut (Rawls 2018). Folglich muss auch die Analyse den Prozesscharakter der Situationsdefinition berücksichtigen.

Einen wichtigen Aspekt für das Gelingen der ethnografischen Beobachtung stellt die im Feld eingenommene Beobachterrolle dar. Das Ziel war hier, eine Position zu finden, welche es ermöglicht, die organisationalen Prozesse zu überblicken, ohne diese selbst zu stören. Zusätzlich sollte sie die Möglichkeit bieten, Notizen machen und bei Unklarheiten nachfragen zu können. Um eine möglichst lückenlose Dokumentation des Arbeitsalltags gewährleisten zu können, wurden die beobachteten Projektleitenden bei sämtlichen arbeitsrelevanten Tätigkeiten begleitet. Diese Beobachtungsstrategie wird in der Literatur als Shadowing bezeichnet (McDonald 2005). Der Beitrag der ethnografischen Forschungsmethode liegt für das vorliegende Forschungsprojekt darin, Koordinationsmechanismen als Bezug auf Objekte, Formen und Konventionen „at work“ mitverfolgen zu können. Dies ermöglicht es, die Prozessualität der Produktion von Survey-Daten und insbesondere die pragmatische Koordination verschiedener Survey-Welten in einem Survey-Projekt mitverfolgen zu können. 
Eine Bedingung für theoriegeleitetes ethnografisches Forschen ist die permanente Reflexion und Verschriftlichung der beobachteten Situationen. Die permanente Verschriftlichung dient der theoriegeleiteten Reflexion über das untersuchte Forschungsobjekt, das heißt zunächst der theoretischen Einordnung, dann aber auch der theoretischen Weiterentwicklung und Begriffsbildung (Hirschauer 2001). ${ }^{22}$ Dieser Wechsel zwischen Beobachtung und Reflexion kann als going native - coming home beschrieben werden (Breidenstein et al. 2013, S. 42). Phasen des going native und des coming home wechseln sich dabei ständig ab, wodurch ein prinzipiell unendlicher hermeneutischer Zirkel entsteht. Die beiden Forschungsschritte Datenerhebung und Datenauswertung wechseln sich so kontinuierlich ab, wobei sich beide Schritte gegenseitig aufeinander stützen (Gobo 2008, S. 86). Praktisch bedeutet dies, dass nach Phasen der Datenerhebung die in den Abschn. 4.3 und 4.4 dargestellten Techniken der Datenanalyse zum Einsatz kamen und auf Basis von Ergebnissen dieser Analyse weitere Daten erhoben wurden.

\subsection{Identifikation und Analyse von Survey-Welten}

Nach einer ersten umfassenden Datenerhebung stellten die Identifikation und die Analyse der verschiedenen Survey-Welten den zweiten Forschungsschritt dar. Zentral ist hierbei die Erstellung einer Heuristik, welche die theoriegeleitete Analyse des Datenmaterials gewährleisten soll (Diaz-Bone 2015a, S. 328 ff.). Die Analyse des Datenmaterials (Interviews, Gesprächs- und Beobachtungsnotizen sowie Dokumente aus dem Feld wie Richtlinien des VSMS-Verbandes, Zeitungsberichte über das Feld, Homepages der Survey-Firmen, Werbeprospekte der Survey-Firmen, Verträge zwischen Survey-Firmen und Auftraggebern und Projektbeschreibungen) geschah folglich nicht ad-hoc. Sie wurde vielmehr durch eine vorab systematisierte Heuristik im Sinne von theoretischen Codes angeleitet (Strauss 1998, S. 64 f.). Trotz der Codierstrategie mittels soziologisch konstruierter Kodes in der Grounded Theory orientiert sich die Grounded Theory grundlegend an der gegenstandsbezogenen Theoriebildung (Strauss 1998, S. 25). Deswegen wurde die Codierstrategie von Diaz-Bone übernommen, welche Analysestrategien der Grounded Theory mit strukturalistischen Strategien kombiniert

\footnotetext{
${ }^{22} \mathrm{Vgl}$. hierzu auch das Konzept des Memoschreibens in der Grounded Theory (Strauss 1998, S. 175 ff.).
} 
(Diaz-Bone 2010c, S. 197 ff., 2015a). So wurde - im Unterschied zur klassischen Analysestrategie der Grounded Theory - bereits vor der Datenanalyse eine Heuristik erstellt, welche jedoch während des Analyseprozesses auf Basis der Daten ergänzt wurde. Dies hängt unmittelbar mit der methodologischen Position der EC zusammen, welche pragmatische mit strukturalistischen Elementen kombiniert (Diaz-Bone 2018, S. 380 ff.). Aus strukturalistischer Perspektive stellt sich dabei die Anforderung, die richtige Analyseebene zu treffen, nämlich die Ebene der die Praxis strukturierenden Handlungsstrukturen, d. h. Rechtfertigungsordnungen und Konventionen (Diaz-Bone 2010c, S. 322 ff.). Die Analyseheuristik kann sich folglich nicht alleine am empirischen Datenmaterial orientieren, sondern muss die durch die Theorie vorgegebenen Dimensionen der Rechtfertigungsordnungen und Konventionen miteinbeziehen.

Die Arbeit durch den Vergleich stellt in dieser Untersuchung eine zentrale methodische Strategie dar, um die unterschiedliche Logik und Praxis der verschiedenen Produktionswelten aufzeigen zu können und deren Kontingenz sichtbar zu machen. ${ }^{23}$ Verglichen werden in diesem Forschungsschritt weder unterschiedliche Situationen, noch verschiedene Surveys miteinander. Das Ziel ist es vielmehr in (Koordinations-)Situationen (von Survey-Projekten) referenzierte Logiken der Survey-Produktion zu identifizieren. Mit fortschreitender Analyse werden die identifizierten Logiken um verschiedene Dimensionen ergänzt und gegenüber anderen Logiken der Survey-Produktion abgegrenzt. So wird sichergestellt, dass aus der ersten Identifikation von unterschiedlichen Logiken der Survey-Produktion mit fortschreitender Analyse zunehmend umfassende Survey-Welten aus dem Datenmaterial herausgearbeitet werden. Dadurch, dass nicht Situationen oder Surveys selbst das Objekt der Analyse in diesem Forschungsschritt der Identifikation von Survey-Welten darstellen, wird der Einsicht der EC Rechnung getragen, wonach Situationen selbst oft durch eine Pluralität an strukturierenden Logiken gekennzeichnet sind (Boltanski und Thévenot 2007, S. 303 ff.). Das Ziel der Identifikation und der Analyse von Survey-Welten ist es dabei gerade, die in der Praxis geschlossenen Kompromisse zu entwirren und die dabei beteiligten Logiken der Survey-Produktion idealtypisch zu rekonstruieren.

Die verwendete und im Folgenden dargestellte Heuristik wurde maßgeblich aus den Überlegungen von Storper und Salais zu den verschiedenen produktionsweltlichen Konventionen und den sich daraus ergebenden Koordinationsformen

\footnotetext{
${ }^{23}$ Hierin zeigt sich das strukturalistische Erbe der EC. Vgl. hierzu Diaz-Bone (2018, S. $380 \mathrm{ff}$.).
} 
in den verschiedenen Produktionswelten entwickelt (Storper und Salais 1997, S. 26 ff.). Storper und Salais machen beispielsweise deutlich, dass Produkte in den verschiedenen Produktionswelten unterschiedlich definiert werden. Ist in der industriellen Produktionswelt lediglich das „Endprodukt“ der Fertigung das verkaufte Produkt, so ist beispielsweise der Beratungsprozess in der interpersonellen Welt nicht vom „Endprodukt“ zu trennen, da das Produkt nicht ohne die vorherige Feststellung des spezifischen Bedürfnisses möglich ist (Storper und Salais 1997, S. 32 ff.). Zusätzlich machen sie deutlich, dass damit unterschiedliche Arbeitskonventionen und unterschiedliche Akteursformen einhergehen (Storper und Salais 1997, S. 59). Storper und Salais machen auch deutlich, dass verschiedene Produktionswelten Innovationen auf unterschiedliche Art und Weise hervorbringen und folglich auch neue Technologien auf unterschiedliche Weise in Arbeitsprozesse einbinden (Storper 1996; Storper und Salais 1997, S. 63 ff.). Entsprechend leitete die Theorie der Produktionswelten die heuristischen Fragestellungen zu den Zielen von Surveys, zur Produktdefinition, zu typischen produktionsweltlichen Herausforderungen, zur Konkurrenz zwischen Produktionswelten, zur Reaktion von Produktionswelten auf neue Technologien, zur Definition von „guten“ Käufern und Verkäufern, zum typischen Verhältnis zwischen Käufern und Verkäufern und zum Entstehen von geschäftlichen Beziehungen an. Die Überlegungen von Storper und Salais wurden jedoch in Fragen der Wertigkeit und der Größe durch Überlegungen der Rechtfertigungstheorie von Boltanski und Thévenot ergänzt (Boltanski und Thévenot 2007, S. 108 ff.). Zentrale Aspekte der Rechtfertigungstheorie für die vorliegende Untersuchung sind das Aufzeigen von unterschiedlichen Rechtfertigungsordnungen, damit einhergehende unterschiedliche Logiken der Qualitätsbewertung und insbesondere auch unterschiedliche Modelle der Prüfung (Boltanski und Thévenot 2007, S. 200). Aus der Rechtfertigungstheorie abgeleitete Fragestellungen der folgenden Heuristik betreffen die Frage nach unterschiedlichen Qualitätskriterien in den verschiedenen Produktionswelten, nach der Art und Weise von Qualitätstests, der Transformation von „Grösse“ zwischen verschiedenen Welten, nach dem Investitionsmodus von Projektleitenden (um Größe zu erlangen, vgl. Boltanski und Thévenot 2007, S. 199) und nach dem Bewusstsein von Projektleitenden für die Pluralität von Produktionswelten. Die Frage nach der unterschiedlichen Stützung von Produktionswelten durch Formen basiert auf dem Konzept der Forminvestition von Laurent Thévenot (1984). Das Konzept der kognitiven Ambiguität lässt sich ebenfalls der Rechtfertigungstheorie zuordnen und wurde von Laura Centemeri eingeführt (Centemeri, S. 21). Dieses Konzept begründet die Fragestellung nach einer unterschiedlichen kognitiven Ambiguität in den verschiedenen Produktionswelten. Der Fokus auf eine eventuelle Konkurrenz zwischen 
verschiedenen Produktionswelten geht auf die Arbeiten von Pierre Boisard zurück, welcher eine Konkurrenzsituation zwischen verschiedenen Logiken der Käseproduktion identifizierte (1991). Zusätzlich wurden Fragestellungen aus weiteren Theorieansätzen übernommen. So ist die Theorie der Distributed Cognition (Hutchins 1996) und der Global Value Chains (Gereffi et al. 2005) der Ursprung für die Frage nach der systematisch unterschiedlichen Organisation von verschiedenen Produktionsketten. Die den systematischen Vergleich anstoßenden heuristischen Fragen sind:

- Welche Ziele sollen mit den durchgeführten Surveys erreicht werden?

- Was sind zentrale (praktische) Qualitätskriterien für die Produktion von Surveys?

- Was beinhaltet das „Produkt“, welches Survey-Firmen verkaufen?

- Wie werden diese Qualitätskriterien getestet und überprüft?

- Existieren typische Herausforderungen und/oder (ungelöste) Probleme? Wie wird mit diesen umgegangen?

- Stehen die verschiedenen Survey-Welten in Konkurrenz zueinander?

- Gelten gewisse Qualitätsaspekte für mehrere Survey-Welten?

- Wie wird auf neue Technologien reagiert? ${ }^{24}$

- Was sind „gute“ Projektleiter aufseiten der Käufer und aufseiten der Survey-Firma? Was zeichnet diese aus? ? $^{25}$

- Was müssen Projektleitende in einer Produktionswelt leisten/investieren, um „groß“" zu werden?26

- Was sind „gute“ Kunden in einer Produktionswelt? Über welche Fähigkeiten müssen diese verfügen?

- Was ist die typische Beziehung zwischen Käufer und Survey-Firma?

\footnotetext{
${ }^{24}$ Storper und Salais sehen Innovation nicht auf die Welt der intellektuellen Ressourcen beschränkt, sondern betonen, dass Innovation in jeder Produktionswelt stattfindet. Wichtig ist, dass Innovation in Relation zu den Konventionen einer Produktionswelt gesetzt wird (1997, S. 70 ff.). Die Analysedimension der Reaktion auf neue Technologien in der Survey-Forschung zielt gerade auf die Analyse des produktionsweltenspezifischen Umgangs mit Innovation.

${ }^{25}$ Storper und Salais beschreiben unterschiedliche Arbeitskonventionen (1997, S. 57 ff.). Die Bewertung von Arbeit und der Wertigkeit von Projektleitenden in den verschiedenen Survey-Welten geschieht folglich unterschiedlich.

${ }^{26}$ Das Konzept der Größe stammt von Boltanski und Thévenot und wird hier auf die Analyse der Wertigkeit von Projektleitenden in den verschiedenen Survey-Welten bezogen (2007, S. 199).
} 
- Wie entstehen geschäftliche Beziehungen in den verschiedenen Produktionswelten?

- Wie wird mit der kognitiven Ambiguität, d. h. mit dem Wissensvorsprung durch die Survey-Firma im Hinblick auf die Survey-Produktion, umgegangen? Wird/wie wird eine Transparenz der Survey-Produktion hergestellt?

- Welche Formen stützten den Koordinationsprozess zwischen Käufer und Survey-Firma?

- Ist der Koordinationsprozess zwischen Käufer und Survey-Firma unterschiedlich auf Konventionen, Formen, Objekte und Akteure in den verschiedenen Welten verteilt? $?^{27}$

- Besteht aufseiten von Projektleitenden ein Bewusstsein für die Existenz unterschiedlicher Survey-Welten? Wie wird damit umgegangen?

Die Analyse gestaltete sich in der Folge zweiteilig: Einerseits war sie orientiert an der vorgegebenen theoriegeleiteten Heuristik. Andererseits war sie an den Strukturen der Daten orientiert. Aus den Daten wurden neue Codes generiert, die sich als relevant für die Beantwortung der grundlegenden Forschungsfrage nach unterschiedlichen Koordinationslogiken in Survey-Projekten herausstellten, jedoch in der Theorie der Produktionswelten per se nicht vorgesehen sind. Die Einsicht, dass die akademische Survey-Welt fast vollumfänglich in staatlich geförderten Institutionen wie Universitäten oder Hochschulen stattfindet, stellt hierfür ein Beispiel dar. Dies stellt einen Unterschied zur Welt der intellektuellen Ressourcen dar, welche bei Storper und Salais ebenfalls privatwirtschaftlich organisierte Produktionswelten darstellen (Storper und Salais 1997, S. 36 f.). Ein anderes Beispiel stellt die Feststellung der kollektiven Herausforderung von Standards dar. Die Erstellung dieser Standards wird bei Storper und Salais nicht tiefergehend thematisiert, obwohl ihnen ein zentraler Stellenwert in der industriellen Produktionswelt zukommt (Storper und Salais 1997, S. 34) ${ }^{28}$ Bei der Identifikation und Ausarbeitung der Welt der Survey-Information wurde hingegen deutlich, dass dieser Prozess, welcher sich hier in Form der Auswahl und der Definition von Erhebungskategorien zeigt, eine zentrale Herausforderung für

\footnotetext{
${ }^{27}$ Die interpersonelle Welt bei Storper und Salais baut stark auf dem Erfahrungsschatz des Verkäufers auf (1997), während die industrielle Welt beispielsweise auf die Etablierung von Standards, d. h. Formen mit einer hohen Reichweite, angewiesen ist (Storper und Salais 1997, S. 32 f.). Die beiden hier verglichenen Produktionswelten nehmen folglich in unterschiedlicher Weise auf Akteure und Formen Bezug.

${ }^{28}$ Vgl. für eine Theoretisierung von Standards Lawrence Busch (2011).
} 
diese Survey-Welt darstellt. Der fehlende Miteinbezug dieser Herausforderung in die Analyse hätte zur Folge, ein wichtiges Merkmal dieser Survey-Welt nicht zu thematisieren.

Bei der auf den heuristischen Fragen aufbauenden Datenanalyse wurde von der Grounded Theory das Prinzip der wiederholenden Datenanalyse übernommen. Der Datenkorpus wird demzufolge mehrmals durchgearbeitet, da so die Daten durch sich erst aus der Datenanalyse ergebende Codes erneut befragt werden können (Gobo 2008, S. 86; Strauss 1998, S. 46 f.). Zusammengefasst sollte es die hier dargestellte Codierstrategie ermöglichen, die aus der Konventionentheorie abgeleitete Ebene der Konvention zu treffen, gleichzeitig aber den Grundsätzen des methodologischen Experimentalismus (Bogusz 2013) entsprechend der Eigensinnigkeit der Empirie folgen zu können (Blumer 2004, S. 344). Damit wurde auch auf methodischer Ebene ein Kompromiss zwischen den pragmatischen und strukturalistischen Elementen der EC geschlossen (Diaz-Bone 2018, S. 380 ff.).

Als technische Infrastruktur für die Auswertung der Daten (Transkribierte Interviews, Zeitungsartikel, Notizen der ethnografischen Beobachtung, Publikationen der Marktforschung, Homepages etc.) wurde das Datenanalyseprogramm Atlas.ti verwendet. Beachtet werden muss bei der Verwendung dieser Software für Analysezwecke, welche nicht an die Grounded Theory angelehnt sind, die Verankerung von Atlas.ti in dieser Forschungsperspektive (Atkinson 2015, S. 59; Diaz-Bone 2010c, S. 200 f.) Da die dieser Forschungsarbeit zugrunde liegende methodologische Ausrichtung nicht diejenige des Symbolischen Interaktionismus ist, muss entsprechend Abstand genommen werden vom Codierparadigma der Grounded Theory. ${ }^{29}$ An dessen Stelle treten die im Abschn. 4.1 dargestellten methodologischen Grundsätze der Soziologie der Konventionen.

Die Codierstrategie der Grounded Theory unterscheidet zwischen drei verschiedenen Kodierstufen: Dem offenen, dem axialen und dem selektiven Codieren. Das offene Codieren dient dem Aufbrechen des Datenkorpus. Beim

\footnotetext{
${ }^{29}$ Das Kodierparadigma der Grounded Theory stellt dabei in der Grounded Theory selbst das Grundgerüst der folgend beschriebenen Analysestrategie dar und darf nicht mit der Kodierstrategie selbst verwechselt werden. Soziale Phänomene werden hiernach nach den Bedingungen, den Interaktionen zwischen den Akteuren, den Strategien und Taktiken und den Konsequenzen analysiert (Strauss 1998, S. 56 ff.). Dieses (inhaltliche) Grundschema der Analyse ist dabei unmittelbar mit der theoretischen Fundierung der Grounded Theory durch den symbolischen Interaktionismus verknüpft (Diaz-Bone 2010c, S. 200). So wären beispielsweise die „Bedingungen“ sowie die „Strategien und Taktiken“ aus Sicht der EC nach deren konventionellem Fundament zu befragen.
} 
Durchlesen des Datenkorpus werden Textstellen ${ }^{30}$ mit Codes versehen, was den Effekt einer quantitativ zunehmenden Kodifizierung des Datenkorpus zur Folge hat. Der zentrale Analysefokus bildete dabei das Konzept des kompetenten Akteurs, welcher im Engagement mit Objekten, Formen und Konventionen unsichere Situationen koordiniert. Während diesem Schritt wurde versucht, Konzepte zu identifizieren, welche aus der Forschungsperspektive zentral für die Koordination in Survey-Projekten sind. Hier zeigte sich, dass beispielsweise verschiedene Kundengruppen existieren, welche ihre eigenen Ansprüche an eine Survey-Dienstleistung haben, was sich in einer unterschiedlichen Arbeitsorganisation und unterschiedlichen Kompetenzen der Projektleitenden niederschlägt. Diese ersten Konzeptideen wurden in Form von Exposés und Memos verschriftlicht.

Dies stellte bereits den Übergang zum nächsten Codierschritt dar, dem axialen Codieren. ${ }^{31}$ Im Gegensatz zum offenen Codieren wird bei diesem Codierschritt fokussierter auf sich aus den Daten ergebende Konzepte hin codiert. Es geht also weniger um ein quantitativ ausführlicheres Codieren, sondern vielmehr um die Klärung von Beziehungen zwischen Codes, was in der Grounded Theory der Ausarbeitung von Konzepten entspricht (Strauss 1998, S. 101). Im Zentrum dieses Analyseschritts steht die Identifikation und Beschreibung der unterschiedlichen Koordinationslogiken der verschiedenen Survey-Welten. Dies geschah insbesondere durch die Tabellierung und konstante Verschriftlichung verschiedenartiger Ausprägung in den durch die theoriegeleiteten Codes vorgegebenen survey-weltlichen Vergleichsdimensionen (Breidenstein et al. 2013, S. 135). Dabei wurde praktisch mit einem dreistufigen Analysesystem gearbeitet. Codiert

\footnotetext{
${ }^{30}$ Wobei grundsätzlich verschiedene Datenformate in Frage kommen. In der vorliegenden Untersuchung wurden hauptsächlich Textdaten verwendet, aber auch Audiodateien von Interviews. Interviews, bei denen unklar war, inwiefern sie relevant für die Beantwortung des Forschungsinteresses sind, wurden zunächst in Atlas.ti angehört und vereinzelt kodiert. Stellte sich das Interview als relevant für die Beantwortung des Forschungsinteresses heraus, wurde das Interview transkribiert, um eine einfach zu bearbeitende Datengrundlage zu schaffen, welche zusätzlich auch einfacher zugänglich für die Projektmitarbeitenden ist. Einige Interviews wurden jedoch nicht transkribiert, so dass im Datenkorpus auch Audiodateien vorhanden sind, welche für die Analyse jedoch eine untergeordnete Rolle spielen.

${ }^{31}$ Wobei darauf hingewiesen werden muss, dass die Grounded Theory nicht die Idee klar getrennter Analyseschritte verfolgt. Es ist vielmehr geradezu charakteristisch, dass die verschiedenen Analyseschritte - einerseits in der Datenanalyse, andererseits bei der Trennung zwischen Datenanalyse und Datenerhebung - nicht klar getrennt sind (Strauss 1998, S. $52 \mathrm{ff}$.).
} 
wurde mit Atlas.ti. Eine erste Verschriftlichung und die Tabellierungen wurden in einem separaten Forschungsdokument vorgenommen. Mit dem Fortschreiten der Analyse wurden die Ergebnisse schlussendlich immer mehr direkt in der Dissertation selbst verschriftlicht. Durch die Arbeit mit diesem dreistufigen Analysesystem konnte konstant zwischen verschiedenen Analysestufen gewechselt werden.

In einem weiteren Codierschritt - dem selektiven Codieren - sollte die Frage geklärt werden, wie die während des axialen Codierens identifizierten Charakteristika von Survey-Welten der verschiedenen Vergleichsdimensionen untereinander zusammenhängen. Die Daten wurden darum verstärkt auf die sich herausbildenden Schlüsselkonzepte des Wissensformats und der unterschiedlichen Epistemologien hin untersucht. An diesem Punkt war der Miteinbezug der Forschungsperspektive besonders wichtig, um die sich herausbildenden Schlüsselkonzepte konzeptionell durch deren Theoretisierung für den soziologischen Diskurs fruchtbar zu machen. Da wie bereits dargestellt die Analyseschritte der Codierung und der inhaltlichen Beschreibung der Survey-Welten bewusst nicht klar getrennt waren, wurden durch inhaltliche Erkenntnisse weitere Datensammlungen angestoßen. Zum Einsatz kamen hier unterschiedliche Dokumente aus den verschiedenen Produktionswelten (Zeitungsartikel, Publikationen der Marktforschung, Homepages, Richtlinien, Verträge etc.). Auch bei diesem Analyseschritt wurde mit dem dreistufigen Analysesystem Atlas.ti, Forschungsdokument und Arbeit an der Dissertation gearbeitet. Auch bei diesem Analyseschritt wurde mit einer Kombination zwischen der Analyse in Atlas.ti, der konzeptionellen Arbeit mit einem separaten Forschungsdokument und dem Niederschreiben von ersten Textstücken in der Dissertation gearbeitet. Zusätzlich wurde hier verstärkt mit Grafiken als Analyseinstrument gearbeitet. Ein Beispiel hierfür stellt die Erstellung von idealtypischen statistischen Ketten der verschiedenen Survey-Welten oder die Erstellung einer Grafik zum Verhältnis der verschiedenen Survey-Welten zueinander dar.

Folgend soll ein Beispiel einer Identifikation einer Produktionswelt dargestellt werden, um die intersubjektive Nachvollziehbarkeit des Prozesses der Identifikation und Analyse von Survey-Welten ermöglichen zu können. $\mathrm{Zu}$ diesem Zweck wird an den folgenden beiden Interviewausschnitten die grundlegende Interpretationsstrategie dargestellt. In der folgenden Sequenz wurde seitens der Interviewenden darauf hingewiesen, dass es ,von außen“ betrachtet unterschiedliche Ausrichtungen der verschiedenen Survey-Firmen gebe. Die interviewte Person - langjährig bei einer Survey-Firma in einer Führungsposition tätig - erklärt daraufhin zwei unterschiedliche Positionierungen von Firmen: 
Jaja, die verkauf, also [die Konkurrenzfirma] verkauft im Prinzip auch eher fixe Produkte. Im Marketingforschungsbereich. Wogegen wir machen ad-hoc Forschung. Also wir erfinden das Produkt immer neu. Wir verstehen uns eben als Forscher. Und die [Konkurrenzfirma] versteht sich schon eher als Consultant, geht dann mit einem Standard, oft mit einem Standardprodukt zum Kunden geht. Weil die haben [...] Geld, weil standardisierte Produkte. Und die wollen sie zunehmend in allen Ländern verkaufen.

Deutlich werden hier zwei Punkte: Einerseits scheinen zumindest zwei Pole zu existieren im Hinblick auf die Survey-Produktion: Standardisierte versus flexible, entdeckungsorientierte Surveys. Es wird darüber hinaus jedoch klar, dass nebst der Standardisierung auch eine Vergleichbarkeit bei der Konkurrenzfirma identifiziert wird, welche sich an der internationalen Vergleichbarkeit der vertriebenen Produkte zeigt. Die interviewte Person nimmt kurz darauf eine weitere Einteilung der eigenen Positionierung vor:

Jaja, das ist eben, das [Anm.: die Positionierung der Konkurrenzfirma] ist ein bisschen eine andere Philosophie. Und unsere Nische ist eben, dass man bei uns noch etwas [...] Maßgeschneidertes bekommt. Wir haben schon also so Grobkonzepte [...] die wir durchdacht haben, wo wir einfach wissen, das funktioniert so und so. Aber das ist kein fixes Produkt.

Deutlich wird hier, dass die eigene Positionierung einen Kompromiss einnimmt zwischen einer vollständig an die Situation des Kunden angepassten Forschungsstrategie und stärker an einer Standardisierung ausgerichteten Orientierung an „Grobkonzepten“. Dies wurde zum Anlass genommen, nach idealtypischeren Survey-Projekten, Projektleitenden oder Survey-Firmen beider Pole zu suchen, um in einem darauffolgenden Schritt den im Interview genannten Kompromisscharakter in seiner Kompromissproblematik besser verstehen und analysieren zu können. Dieser Kompromisscharakter von Survey-Projekten trat insbesondere in den beiden Fallanalysen zutage. Die idealtypisch identifizierten Survey-Welten halfen hier dann, die Herausforderung der Kombination verschiedener Survey-Welten in einem Projekt analysieren und beschreiben zu können.

Unklar bleibt im zitierten Ausschnitt des Interviews jedoch, inwiefern die Vergleichbarmachung mit der Standardisierung zusammenhängt. Zentral für diese Unterscheidung war der Abgleich mit der Produktionsweltentheorie. Aus dieser Perspektive wird sichtbar, dass der Unterschied zwischen der Vergleichbarmachung und der Standardisierung darin liegt, dass generische Welten - und somit die Vergleichbarmachung - die Produktion ohne Beachtung der individuellen Eigenheiten der Kunden vornimmt. Wie sich später herausstellen sollte, findet bei 
der von der interviewten Person erwähnten Konkurrenzfirma teilweise tatsächlich eine Vorerhebung von Daten vor dem Kontakt mit den einzelnen Kunden statt. Deshalb kann in Bezug auf die Gesamtstrategie von einem Kompromiss zwischen Standardisierung und Vergleichbarkeit gesprochen werden.

Entscheidend für die Identifikation der verschiedenen Survey-Welten war, dass die Empirie Hinweise lieferte durch Abgrenzungen, Kritik und Positionierungen. Diese wurden systematisch zu ordnen versucht, insbesondere durch den Bezug auf Rechtfertigungsordnungen und die Produktionsweltentheorie. Dabei zeigte sich erst nach intensiver Analysearbeit, dass die Produktionsweltentheorie eine starke Erklärungsleistung für die durch die interviewten und beobachteten Personen vorgenommene Differenzierung des Survey-Marktes bot. Das Konzept der Produktionswelten mit seiner relativ starren Einteilung von Differenzierungen von Märkten stand folglich nicht am Anfang der Forschung, sondern hat sich quasi in Ko-Evolution während der empirischen Analyse durchsetzen können. ${ }^{32}$

\subsection{Fallanalyse}

In diesem Unterkapitel wird die Vorgehensweise der fallbasierten Analyse beschrieben. Im Gegensatz zur Identifikation von Survey-Welten ist es hier nicht das Ziel, Survey-Welten idealtypisch zu rekonstruieren, sondern eine Analyse von beobachteten Survey-Projekten im Hinblick auf deren survey-pragmatische und survey-weltliche Koordination vorzunehmen. Fälle sind hierbei statistische Ketten von Survey-Projekten, d. h. der Prozess der Survey-Produktion über verschiedene Stationen und Situationen hinweg. Survey-Projekte sind dabei in der Regel durch einen Kompromiss zwischen verschiedenen Survey-Welten gekennzeichnet. ${ }^{33}$ Das Ziel der Fallanalyse liegt entsprechend im Nachzeichnen der

\footnotetext{
${ }^{32} \mathrm{Zu}$ Beginn bestand die Idee, Produktionswelten durch deren starken Fokus auf die transorganisationale Koordination als Konzept zu verwenden, diese jedoch eng mit dem Konzept der Rechtfertigungsordnungen zu verbinden, da zu diesem Zeitpunkt die Auftrennung des Survey-Marktes in die Pole generisch - gewidmet und standardisiert - unstandardisiert als unplausibel erschien.

${ }^{33}$ Survey-Projekte, welcher nur einer Welt zugeordnet werden können, sind durchaus denkbar, jedoch gerade durch den in der Schweiz in den allermeisten Fällen notwendigen Rückgriff auf Survey-Firmen auch durch akademische und amtliche Surveys nicht die Regel. Die von Storper und Salais rekonstruierten realen Produktionswelten sind ebenfalls durch einen Kompromiss aus verschiedenen möglichen Produktionswelten gekennzeichnet (1997, S. 97 ff.).
} 
handlungspragmatischen, situativen Herausforderungen, welchen sich Projektleitende in Survey-Projekten gegenüber konfrontiert sehen, die durch Kompromisse zwischen verschiedenen Produktionswelten entstehen und welche sie durch den Bezug auf Objekte, Formen und Konventionen kompetent zu lösen versuchen. Durch die Fallanalysen wird die Einlösung der pragmatischen Perspektive der Soziologie der Konventionen angestrebt, sodass nicht nur Konventionen als Koordinationsgrundlagen von Survey-Projekten herausgearbeitet werden, sondern ebenfalls deren pragmatische Handhabung in handlungsoffenen Situationen. Der Anspruch der Fallauswahl liegt nicht in einer Repräsentativität der Fälle für ein erforschtes Feld begründet, sondern im Auffinden, Beschreiben und Analysieren von generalisierbaren Prozessen und Mechanismen (Flick 2009, S. 260).

Das Fallkonzept ist folglich ein offenes Forschungskonzept, dessen inhaltliche Ausgestaltung während des Forschungsprozesses aus dem Material herausgearbeitet werden muss. Denn Fälle beziehen sich nicht auf eine vor der Forschung identifizierbare Kultur oder Gruppe, sondern auf soziale Prozesse (Mitchell 2006). In der vorliegenden Arbeit sind Fälle statistische Ketten von Survey-Projekten, in welchen die Produktion von statistischen Daten koordiniert wird. Das Ziel der Fallbeschreibungen liegt nicht in einer Nacherzählung des Vorgefallenen, bzw. Gesagten (Hammersley und Atkinson 2007, S. 159), sondern in einer analytischen Aufarbeitung einzelner Situationen von Survey-Projekten. Eine analytische Aufarbeitung eines Falls meint dabei, eine auf Basis der Forschungsperspektive vorgenommene Interpretation der Prozesse und Koordinationsleistungen vorzunehmen. Es soll bei diesem Punkt folglich darum gehen, über das Engagement von kompetenten Akteuren unter dem Einbezug von Objekten, Formen und Konventionen zu reflektieren. Trotz der Falleingrenzung fokussiert die Fallanalyse nicht auf lokale Gegebenheiten, sondern zieht weitreichende Phänomene mit in die Analyse ein - sofern auf sie durch kompetente Akteure Bezug genommen wird.

Die survey-methodologischen Überlegungen aus Kap. 2 zusammen mit den Experteninterviews waren die Grundlage für eine theoriegeleitete Fallauswahl für die ethnografischen Beobachtungen im Sinne eines "theoretical samplings“ (Strauss 1998, S. 70 f.). Das theoretical sampling stellte die Grundlage für die Durchführung weiterer Interviews und die konstante Generierung von zusätzlichem Datenmaterial dar, welches für die Beantwortung der Fragestellung relevant ist. Da die Datenerhebung nur einen kleinen Teil der relevanten Situationen eines Survey-Projekts einfangen kann, werden auch bei der Fallanalyse ausgewählte Situationen (insbesondere Koordinationssitzungen, aber auch Interviews zu Survey-Projekten) mit weiteren Materialen (Forschungsberichte, Verträge, Projektplanungen, Werbematerial etc.) ergänzt, um der Analyse eine höhere 
Reichweite zu ermöglichen. Zusätzlich zur beschriebenen Codierstrategie kommt hier die Strategie des sequenziellen Durcharbeitens von verschiedenen Situationen eines Survey-Projekts zur Anwendung, in denen das Prozessieren von Survey-Projekten rekonstruiert wird. Die in den vorherigen Abschnitten dargestellte Analysestrategie der Codierung ist für die Fallanalyse nur bedingt geeignet. Denn zumindest das offene Codieren befördert zunächst eine Entkontextualisierung von Daten, während bei der Fallanalyse gerade die Kontextualität und die Prozessualität des Engagements im Zentrum der Analyse stehen. Das Ziel des sequenziellen Durcharbeitens des Datenmaterials liegt in einer Schritt für Schritt-Rekonstruktion von Interviewtranskripten und ethnografischen Beschreibungen. Im Gegensatz zur Codierung geht es jedoch nicht um die Rekonstruktion von möglichst idealtypischen Beispielen für eine Survey-Welt, sondern es ist hier das Ziel, das ,doing“ von Survey-Koordination mit der inhärenten Ambiguität und auf Objekte, Formen und Konventionen Bezug nehmenden prozessualen Engagements zu rekonstruieren. Dabei wird gemäß der Maxime der Voraussetzungsarmut gearbeitet (Knoll 2012, S. 101). Element einer Situation ist folglich nur, was in dieser selbst thematisiert und als Element durch Akteure eingeführt wird. Die erarbeiteten Theoriegrundlagen der Soziologie der Konventionen dienen bei der Fallanalyse als sensibilisierende Konzepte für die Rekonstruktion von Koordinationssituationen (Blumer 1954).

Die zentrale methodische Strategie für die Umsetzung der sequenziellen Analyse von Fällen stellt das Erstellen von Situationsmaps dar. Die Strategie des Mappings hat seinen Ursprung in der Chicago School und erfuhr eine konstante Weiterentwicklung. Die bis jetzt konsequenteste Weiterentwicklung von Maps als sozialwissenschaftlicher Forschungsstrategie stammt von Adele Clarke (Clarke 2012). Gemäß ihr ist die Grounded Theory teilweise noch in positivistischem Denken verankert. Das Ziel der von ihr ausgearbeiteten Situationsanalyse liegt in einer postmodernen Öffnung der Grounded Theory (Clarke 2012, S. 25 ff.). $\mathrm{Zu}$ diesem Zweck integriert sie einerseits die Soziologie von Bruno Latour in die Analysestrategie der Grounded Theory und andererseits die Diskurstheorie von Michel Foucault. Das Ziel der Integration von Latours Soziologie liegt in einer Stärkung des Objektfokus der Grounded Theory. Die Integration der foucaultschen Diskurstheorie dient dem Einbezug von Diskursformationen und insbesondere der Darstellung der Diversität von verschiedenen Diskurspositionen, sowie der Integration von diskursiven Machtphänomenen in die Grounded Theory (Clarke 2012, S. 169 ff., 193 ff.). Den Kernpunkt der Methode der Situationsanalyse von Clarke stellen die verschiedenen Mapping-Strategien dar. Das Ziel der Mappings stellt die Unterstützung der analytischen Aufarbeitung von Situationen dar. Clarke unterscheidet drei verschiedene Mapping-Strategien: Situations-Maps, 
Maps von sozialen Welten/Arenen und Positions-Maps. Situations-Maps dienen bei Clarke einer analytischen Aufarbeitung der verschiedenen Situationselemente einer Situation. Sie dienen folglich einem Aufbrechen der Situation in die verschiedenen in ihr existierenden Situationselemente, aber auch einem ersten Ordnen der Relationen zwischen den verschiedenen Elementen (Clarke 2012, S. 126 ff.). Maps von sozialen Welten/Arenen dienen der Aufarbeitung unterschiedlicher Diskurse. Clarke verknüpft hierbei die Theorie der sozialen Welten von Anselm Strauss (Strauss 1978, 1982, 1984) mit der Diskurstheorie von Foucault (Foucault 2008). Arenen stellen dabei den Integrations- bzw. Konfliktort der verschiedenen weltenbasierten Diskurse dar. In Positionsmaps werden schlussendlich die verschiedenen identifizierten Diskurse/soziale Welten im Hinblick auf ihre diskursive Position systematisiert. Diskurspositionen werden in diesem Schritt anhand verschiedener Diskursdimensionen geordnet. Dies dient einerseits dem Aufzeigen von Übereinstimmungen und Unterschieden von verschiedenen Diskursen, zusätzlich aber auch dem Aufzeigen von fehlenden diskursiven Positionen, was ein Hinweis auf diskursive Macht sein kann (Foucault 2008, S. 169 ff.).

Die Situationsanalyse von Clarke teilt mit der EC zwei grundlegende sozialtheoretische Positionen. Einerseits weist sie ebenfalls ein starkes pragmatisches Element durch ihre Verankerung in der Grounded Theory auf. Andererseits übernimmt sie strukturalistische Konzepte wie die Diskurstheorie durch den Rückgriff auf Foucault. Es ist diese sozialtheoretische Übereinstimmung, welche die Mapping-Strategien gemäß der Situationsanalyse von Clarke zu einer gewinnbringenden methodischen Strategie auch für EC-basierte Forschungsprojekte werden lässt. Eine weitere Gemeinsamkeit zwischen der methodologischen Position der EC und der Situationsanalyse von Clarke stellt der methodologische Fokus auf Situationen dar. Der Untersuchungsfokus ist folglich nicht auf Organisationen oder andere sozialwissenschaftliche Einheiten gerichtet, sondern auf die Situation als integrierenden Ort von Akteuren, Objekten und unterschiedlichen diskursiven Logiken. Die im Folgenden dargestellten, den Fallanalysen zugrunde liegenden Mapping-Strategien folgen der Aufteilung der Mapping-Strategie von Clarke. Diese strebt zuerst eine Identifikation der zu untersuchenden Situation und der unterschiedlichen Situationselemente und anschließend einen Überblick über die verschiedenen in der Situation existierenden sozialen Logiken an. Soziale Logiken werden bei Clarke in Form sozialer Welten thematisiert. Darüber hinaus bestehen jedoch zwischen den von Clarke beschriebenen und der bei der Fallanalyse zur Anwendung gekommenen Mapping-Strategien auch grundlegende Unterschiede. Das grundlegende Ziel der Mapping-Strategie von Clarke liegt in 
der Darstellung und im Beschrieb einer Situation (ebd.: S. 123 ff.). ${ }^{34}$ Das Ziel der hier durchgeführten Fallanalysen liegt zwar auch in der Analyse von Situationen, darüber hinaus aber gerade auch in der Darstellung der Verkettung verschiedener Situationen und dem Einfluss dieser Verkettung auf den Prozess der Survey-Produktion. Zudem liegt der Fokus der Fallanalysen nicht allein auf einem Beschrieb von verschiedenen Situationen, sondern in einer Rekonstruktion der situativen Handlungspragmatik durch die beteiligten Akteure und deren Mobilisierung von Objekten, Formen und Konventionen.

Wie dargestellt, sind „Fälle“ für das vorliegende Forschungsprojekt Prozesse der Survey-Produktion über verschiedene Stationen und Situationen hinweg. Das Ziel der Fallanalyse liegt folglich im Beschrieb und in der Analyse dieser distribuierten Prozesse der Survey-Koordination. Im Hinblick auf das Einsetzen von Mappings können zwei grundlegende Strategien unterschieden werden: Situations- und Koordinationszahl. Situationsmaps zielen auf eine Analyse der verschiedenen in einem Survey-Prozess identifizierten Situationen. Wie in Abschn. 3.1 dargestellt, beschränkt sich das Situationskonzept der EC nicht auf face-to-face Situationen. Der Situationsbegriff ist in der EC ein analytisches Konzept, welches Orte der Koordination zwischen Akteuren, Objekten, Formen und Konventionen beschreibt (Diaz-Bone 2018, S. 374 ff.). Situationen lassen sich folglich analytisch voneinander unterscheiden, wenn in ihnen unterschiedliche Situationselemente koordiniert werden. Das Ziel von Situationsmaps liegt folglich in einer Analyse der verschiedenen Situationselemente, d. h. von Akteuren, Objekten, Formen und Konventionen. ${ }^{35}$

Die methodologische Position der EC betont die Prozesshaftigkeit des Sozialen (Diaz-Bone 2018, S. 328). Das Ziel der zweiten Mapping-Strategie, der Koordinationsmaps, liegt deswegen in einer Rekonstruktion des Prozesses der Survey-Produktion. Das Ziel dieser zweiten Mapping-Strategie ist es, die Verkettung der verschiedenen identifizierten und rekonstruierten Situationen nachzuzeichnen. Das Ziel liegt folglich darin, die Verkettung von Situationen durch deren Bezug auf vor- oder nachlaufende (d. h. erwartete) Situationen darzustellen und dadurch den Survey-Prozess als Abfolge verschiedener Koordinationssituationen zu rekonstruieren. Koordinationszahl orientieren sich dabei wiederum

\footnotetext{
${ }^{34}$ Der Situationsbegriff bei Clarke ist dabei - analog zum Situationskonzept der EC - nicht auf face-to-face-Situationen beschränkt und kann weite raumzeitliche Ausdehnungen annehmen (2012).

${ }^{35}$ Was Konventionen im Sinne von Dodier (1994) oder Favereau (2008) und auch Rechtfertigungsordnungen im Sinne von Boltanski und Thévenot (2007) miteinschließt.
} 
an den verschiedenen dargestellten Situationselementen und analysieren deren Koordination über verschiedene Situationen hinweg. Hier wird folglich analysiert, wie Situationselemente einer Situation durch nachlaufende Situationen thematisiert und koordiniert werden.

In den folgenden Absätzen werden nun die verschiedenen Schritte der Fallanalyse erläutert, d. h. es werden die bei der Auswertung des fallbasierten Datenmaterials zur Anwendung gekommenen Strategien dargestellt. Der erste Schritt der Fallanalyse besteht in der Identifikation von für das eigene Forschungsprojekt geeigneten Fällen. Die Fallauswahl wird deswegen bereits zur Fallanalyse hinzugerechnet, da hier bereits maßgeblich die Möglichkeiten der Fallanalyse vorbereitet werden. Die Fallauswahl hat folglich einen starken Einfluss auf das analytische Potenzial der Fälle. Zwei Kriterien muss ein Survey-Projekt hierfür minimal entsprechen: Zunächst muss es ein Survey-Projekt sein, welches in Zusammenarbeit mit einer Survey-Firma durchgeführt wird. Die Zusammenarbeit mit einer Survey-Firma macht es notwendig, die verschiedenen Anforderungen und Qualitätskriterien eines Surveys stärker zu explizieren, als dies bei einem Survey in Eigenregie notwendig wäre. Dies ermöglicht eine bessere Beobachtbarkeit. ${ }^{36}$ Zudem müssen mehrere Situationen der Survey-Produktion beobachtbar sein, um die Pragmatik von Survey-Projekten im Zeitverlauf analysieren zu können. Das Ziel liegt darin, einerseits diese koordinative Pragmatik anhand einzelner Situationen der Survey-Produktion detailliert herausarbeiten und andererseits diese über den Zeitverlauf des Survey-Projektes systematisieren zu können.

Ein zentrales Kriterium bei der Fallauswahl stellt auch das Potenzial der beiden Fälle einer produktionsweltlichen Kontrastierung dar. Denn das Ziel bei der Fallanalyse besteht gerade darin, die enge Verbindung zwischen unterschiedlichen Survey-Qualitäten und der Survey-Koordination aufzuzeigen. Entsprechend ist es das Ziel, Survey-Projekte zu analysieren, welche in unterschiedlichen Survey-Welten lokalisiert werden können. Denn nur durch den systematischen Vergleich zwischen der Survey-Praxis verschiedener Survey-Welten kann mit der unmittelbaren Angemessenheit der Survey-Praxis gebrochen werden. Die Analyse von kontrastierenden Fällen entspricht folglich einer Strategie des Bruchs, welche die Kontingenz der survey-weltlichen Praxis aufzeigen soll (Bourdieu et al. 1991, S. 60). Obwohl verschiedene Survey-Projekte Teil des Untersuchungskorpus waren, entsprachen schlussendlich zwei Survey-Projekte

\footnotetext{
${ }^{36}$ Durch die Zusammenarbeit von akademischen und amtlichen Survey-Projekten mit kommerziellen Survey-Firmen wird diese Forschungsstrategie erst möglich.
} 
den dargestellten Kriterien. Beide Survey-Projekte müssen dabei Kompromisse zwischen verschiedenen Survey-Welten herstellen. In Fall (A) wird maßgeblich ein Kompromiss zwischen der Beratungs- und der Informationswelt hergestellt, jedoch tauchen in der Projektkoordination auch dienstleistungsweltliche Aspekte auf. Fall (B) ist klar an der akademischen Welt orientiert - es werden hier Daten für die sozialwissenschaftliche Forschung produziert. Es wird jedoch an verschiedenen Stellen des Survey-Projekts deutlich, dass Kompromisse mit der Beratungswelt, der Dienstleistungswelt und auch der Informationswelt geschlossen werden müssen. Die beiden Survey-Projekte stellen folglich dadurch im Hinblick auf ihre survey-weltliche Orientierung kontrastierende Fälle dar, da der jeweilige Hauptfokus der Survey-Projekte in unterschiedlichen Survey-Welten liegt. Obwohl in beiden Survey-Projekten Kompromisse mit anderen survey-weltlichen Konventionen geschlossen werden, so unterscheiden sich die beiden Fälle auch in den dabei zur Anwendung gekommenen Strategien und auftretenden Problemen. Die beiden Fälle kontrastieren sich folglich sowohl im Hinblick auf ihre survey-weltliche Verankerung wie auch in ihrer Kompromissfindung mit anderen Survey-Welten.Im zweiten Forschungsschritt werden die verschiedenen Situationen des Falls identifiziert. Dies sind zunächst Situationen eines Falls, an welchen selbst beobachtend teilgenommen wurde. Innerhalb dieser Situationen wird jedoch auf weitere Situationen verwiesen (wie z. Bsp. auf vorlaufende Besprechungen zwischen Auftraggeber und Survey-Firma, geplante Präsentationen der Resultate, organisationsinterne Besprechungen des Survey-Projekts, verschiedenartige Regelwerke etc.). Diese vor- und nachlaufenden Situationen können einen Einfluss auf die untersuchte Situation haben und sind Teil des Entstehungsprozesses des Survey-Projekts. Anhand einzelner untersuchter Situationen des Survey-Projektes lassen sich in der Folge zunächst auf einzelne Situationen fokussierende Situationsmaps erstellen, welche die untersuchte Situation als Koordination, bzw. Vernetzung verschiedener Situationen darstellen und verstehen lässt. Identifiziert wird hier die Ausstattung der Situation mit Akteuren, Objekten, Formen und Konventionen. Analysiert wird dabei deren situative Mobilisierung, sowie deren Herkunft in anderen Situationen. Mit dem Voranschreiten der Analyse werden weitere identifizierte Situationen der SurveyProduktion analysiert und in die Situationsmaps miteinbezogen. Dabei wird nach vor- und nachlaufenden Situationen unterschieden. Durch die graphische Darstellung entsteht so mit der Zeit eine Map des Survey-Prozesses, in welchem die verschiedenen Koordinationssituationen dieses Prozesses nach deren chronologischem Ablauf dargestellt werden.

Im Analyseschritt drei wird die situationsübergreifende Pragmatik von Survey-Projekten im Hinblick auf verschiedene Analysedimensionen mittels 
des Erstellens von Koordinationsmaps identifiziert. Im Hinblick auf die verschiedenen Dimensionen wird deren Festlegung im entsprechenden Survey-Projekt über mehrere Situationen hinweg nachgezeichnet. Von Interesse sind dabei aber auch Inkohärenzen in der Etablierung der Schlüsseldimensionen, bzw. eine fehlende Festlegung derselben. Für jede Schlüsseldimension der Analyse wurde eine eigene Map angefertigt. Diese Koordinationsketten werden grafisch in die Situationsmaps eingezeichnet, um einen Überblick über zentrale Koordinationssituationen für die jeweilige Schlüsseldimension $\mathrm{zu}$ erhalten. Dies ermöglicht einen systematischen Überblick über das Zustandekommen einer Schlüsseldimension. Die verschiedenen Situationen werden auf die Festlegung folgender Schlüsseldimensionen von Surveys hin befragt: ${ }^{37}$

- Ziel des Surveys (Was soll mit dem Survey erreicht werden?)

- Auswahl der Survey-Firma (Wie und nach welchen Kriterien wird eine Survey-Firma für die Befragung ausgewählt?)

- Definition der Grundgesamtheit (Wie wird diese definiert?; Welche Personengruppen sollen inkludiert werden?)

- Stichprobenziehung (Wie wird die Stichprobe gezogen?)

- Preisfestlegung (Wie wird der Preis für das „Produkt“ festgelegt?)

- Wahl der Befragungsart (Welcher Mode oder welche Mode-Kombination wird gewählt?) ${ }^{38}$

- Fragebogenerstellung (Wie werden die Fragebogenkategorien und das Design des Fragebogens festgelegt?)

- Pretest (Wird der Fragebogen getestet? Durch wen? Wie?)

\footnotetext{
${ }^{37}$ Die Definition der Schlüsseldimensionen geschah auf Basis des von Biemer und Lyberg dargestellten Survey-Prozesses (2003, S. 27). Der Bezug darauf mag zunächst erstaunen, da sich die Kritik an der Prozessperspektive maßgeblich auf die Arbeiten von Biemer und Lyberg bezog (vgl. Abschn. 2.4 und 2.5). Die Kritik an der Prozessperspektive richtete sich jedoch an deren Konzeptualisierung von Survey-Qualität aus. Die Charakterisierung der Schlüsseldimensionen durch Biemer und Lyberg wurde als Ausgangspunkt genommen für die empirische Untersuchung der statistischen Ketten der verschiedenen Survey-Welten. Hierbei zeigten sich unterschiedliche Schwerpunktsetzungen und Abläufe in einigen Survey-Welten im Vergleich zu dem durch Biemer und Lyberg beschriebenen Survey-Prozess. Die Unterschiede und die damit einhergehenden Konsequenzen für die Survey-Methodologie werden in Abschn. 8.1.1 diskutiert.

${ }^{38}$ Ein „Mode“ bezeichnet in der Survey-Methodologie die Befragungsart. Schnell unterscheidet zwischen persönlichen, postalischen, telefonischen und webbasierten Befragungen (Schnell 2012, S. 187 ff.).
} 
- Planung der Feldphase (Wer plant die Detailabläufe der Befragung?; Wer führt sie durch?)

- Daten- und Wissensübermittlung an Auftraggeber (Wie werden/wie wird die erhobenen Daten der Survey-Firma/der Abschlussbericht an die Auftraggeber übermittelt?)

- Datenanalyse (Wie werden Daten analysiert? Was sind hierbei die leitenden Forschungsfragen?)

- Verwendung der Daten (Für welche Zwecke werden die Daten verwendet?; Welchem Publikum werden die Daten präsentiert?)

In Analyseschritt vier werden die Ergebnisse der Analyse der verschiedenen Schlüsseldimensionen aufgenommen. In einer Gesamtschau werden in diesem Schritt die verschiedenen Koordinationsketten der dargestellten Schlüsseldimensionen miteinander verglichen und es wird eine Diagnose des Falls im Hinblick auf dessen Einbettung in Survey-Welten und Konventionen vorgenommen. Geklärt werden soll, welche Einbettungen anhand der über verschiedene Situationen hergestellten Festlegung der verschiedenen Schlüsseldimensionen ersichtlich sind. Damit einhergehend wird der Fall auf zentrale Kompromisse zwischen verschiedenen Produktionswelten und Konventionen befragt. Zentrale Fragestellungen dieses Schrittes bei der Analyse der verschiedenen Schlüsseldimensionen der Survey-Projekte sind:

- Wo zeigt sich der Bezug auf survey-weltliche Konventionen der Survey-Forschung?

- Existiert ein einheitlicher Bezug auf survey-weltliche Konventionen im Hinblick auf die verschiedenen Schlüsseldimensionen?

- Existieren Kompromisse zwischen verschiedenen survey-weltlichen Konventionen?

- Wie werden Kompromisse zwischen survey-weltlichen Konventionen etabliert? Wie wird mit den daraus entstehenden Spannungen umgegangen?

- Lassen sich survey-pragmatische Spannungen durch den Bezug auf bestimme survey-weltliche Konventionen identifizieren? ${ }^{39}$

\footnotetext{
${ }^{39}$ D. h. Konflikte zwischen den verschiedenen Dimensionen der Survey-Pragmatik, d. h. der Methodik, dem Survey-Management und dem angestrebten Wissensformat. Vgl. Abschn. 2.5.5.
} 


\section{1.) Identifikation von Fällen}

\section{2.) Erstellen von Situationsmaps}

- Identifikation von Koordinationssituationen der Surveyproduktion

- Identifikation von Situationselementen (Akteure, Objekte, Formen, Konventionen)

- Erstellen von Situationsmaps, welche nach dem chronologischen Ablauf der verschiedenen Koordinationssituationen ausgerichtet sind.

\section{3.) Erstellen von Koordinationsmaps}

- Analyse der transsituationalen Koordinationsketten zentraler Dimensionen des Survey-Prozesses

- Analyse der Mobilisierung von Akteuren, Objekten, Formen und Konventionen in den identifizierten Koordinationsketten

4.) Verdichtung der Ergebnisse aus Schritt 2 bis 3

- Vergleich der verschiedenen Koordinationsketten eines Falls

- Identifikation der konventionellen und survey-weltlichen Einbettung eines Falls

- Gesamtschau der survey-weltlichen Pragmatik des Falls

5.) Vergleich von Fällen

- Vergleich der Fälle im Hinblick auf ihre konventionelle und survey-weltliche Einbettung

- Interpretation der Survey-Pragmatik, d.h. des Kompromisses zwischen der Methodik, dem Survey-Management und dem Wissensformat der beiden Fälle

Abb. 4.1 Schritte der Fallanalyse

Im letzten Analyseschritt werden die verschiedenen untersuchten Fälle miteinander verglichen. Analysiert werden hier Ähnlichkeiten und Unterschiede der Fälle. Zentral ist die Verortung der Survey-Projekte in Survey-Welten. Analysiert wird hier, wie Eigenheiten der beiden Fälle durch deren Einbettung in unterschiedliche Survey-Welten erklärt werden können. Geklärt wird während dieses Analyseschritts insbesondere auch der Einfluss der survey-weltlichen Verankerung auf die restlichen Dimensionen der Survey-Pragmatik, d. h. die Methodik und das Survey-Management des Surveys.

In Abb. 4.1 sind die verschiedenen gerade beschriebenen Forschungsschritte der Fallanalyse verdichtet dargestellt. 
Open Access Dieses Kapitel wird unter der Creative Commons Namensnennung 4.0 International Lizenz (http://creativecommons.org/licenses/by/4.0/deed.de) veröffentlicht, welche die Nutzung, Vervielfältigung, Bearbeitung, Verbreitung und Wiedergabe in jeglichem Medium und Format erlaubt, sofern Sie den/die ursprünglichen Autor(en) und die Quelle ordnungsgemäß nennen, einen Link zur Creative Commons Lizenz beifügen und angeben, ob Änderungen vorgenommen wurden.

Die in diesem Kapitel enthaltenen Bilder und sonstiges Drittmaterial unterliegen ebenfalls der genannten Creative Commons Lizenz, sofern sich aus der Abbildungslegende nichts anderes ergibt. Sofern das betreffende Material nicht unter der genannten Creative Commons Lizenz steht und die betreffende Handlung nicht nach gesetzlichen Vorschriften erlaubt ist, ist für die oben aufgeführten Weiterverwendungen des Materials die Einwilligung des jeweiligen Rechteinhabers einzuholen.

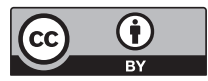

